\title{
RESEARCH
}

Open Access

\section{Study of economic management forecast and optimized resource allocation based on cloud computing and neural network}

\author{
Pinzhen He
}

Correspondence: Pz544871813@163. com

University of York, York YO 105DD, UK

\begin{abstract}
As various factors which affect the development of global market economy become increasingly uncertain, economy and commodity will become more and more fluctuating in economic operation. With its strong nonlinear mapping capacity, artificial neural network has already been applied in many fields, time series analysis, and trend prediction. Cloud computing can interact fast with service provider at the minimum management cost. This paper proposes an economic forecast and optimized resource allocation model based on cloud computing and BP neural network. Its main goal is to break down a complex prediction task into several subtasks, effectively reduce the workload of a single computer and enhance the operating efficiency. Simulation results show that the proposed method does not rely on gradient information and has strong optimization calculation ability. At the same time, it can analyze and predict economic management, so as to provide strong decision support for decision makers.
\end{abstract}

Keywords: Economic management, BP neural network, Cloud computing, Adaptive learning

\section{Introduction}

With more and more uncertain factors that affect the market economy worldwide, the fluctuation of economy and commodity will become increasingly significant in economic operation; so it is necessary to study relevant methods to predict the future trend of economy or commodity and how to optimize and allocate resources. At present, the frequently used economic forecasting system is operating on the centralized platform of a single small-size computer, which has several problems when being used. Besides, different economic indicators use different prediction models and the results obtained are also different [1]. Government decision-makers don't know in advance which prediction model to adopt in order to get the optimal result; so they can only select a model for prediction based on their subjective feeling and the result may not be the best. However, loud-based economic forecasting system can automatically combine prediction indicators with all prediction models, predict every possibility, and automatically simulate and analyze the prediction result and the actual conditions in order to choose the optimal

(c) The Author(s). 2020 Open Access This article is licensed under a Creative Commons Attribution 4.0 International License, which permits use, sharing, adaptation, distribution and reproduction in any medium or format, as long as you give appropriate credit to the original author(s) and the source, provide a link to the Creative Commons licence, and indicate if changes were made. The images or other third party material in this article are included in the article's Creative Commons licence, unless indicated otherwise in a credit line to the material. If material is not included in the article's Creative Commons licence and your intended use is not permitted by statutory regulation or exceeds the permitted use, you will need to obtain permission directly from the copyright holder. To view a copy of this licence, visit http://creativecommons.org/licenses/by/4.0/. 
prediction [2]. With its large-scale parallel structure and distributed storage, neural network has not only strong capacities of function approximation and pattern classification, but also extraordinary self-organization, adaptivity, and fault tolerance, making it very fit for handling practical engineering problems. As massive economic data consistently increase, the computing power of a single microcomputer will eventually become saturated. The continuously expanded capacity of storage devices will increase data transmission time and cost between new devices, which is very uneconomical. On the contrary, neural network which combines cloud environment and adaptive learning can avoid such problem. This combination can fully exert the self-organization, self-learning, and high-fault tolerance so that it can have unique performance and computing power in solving complex nonlinear problems $[3,4]$.

The special contributions of this paper include the following:

- This paper studies the features, methods, and steps of economic management forecast, including qualitative and quantitative prediction, computation, and analysis of prediction error.

- This paper analyzes cloud-based economic forecasting system, including the construction of the overall architecture, cloud client, the maintenance of backend data, and display function.

- This paper studies the optimized structure of BP neural network, analyzes the optimization extent of network structure by using secondary descent and gradient descent, as well as the law of error change, and adopts adaptive learning rate to increase the number of neurons in hidden layer or the layers of network so as to get a proper neural network structure.

- This paper presents a BP neural network based on cloud computing and momentum-adaptive learning rate, realizes economic forecast and optimum allocation of resources, makes simulation experiment, and conducts analysis of the result. As shown in the result of the simulation experiment, this method can find a feasible solution within the designated generalization ability and approximate error range reliably and effectively.

The rest of this paper is organized as follows. Section 2 discusses related works, and cloud-based economic forecast framework is outlined in Section 3. Economic prediction and optimized resource allocation of BP neural network based on momentum-adaptive learning rate is presented in Section 4. Section 5 shows the experimental test results, and Section 6 concludes the paper with a summary and proposed directions for future research.

\section{Related work}

Cloud computing is the development of distributed computing, parallel computing, and grid computing. It has scale effect, service virtualization, shared resource pool, fast and flexible deployment, and on-demand services. Cloud computing has a pretty large scale, and it allows its users to get service at any place with any terminal. The resource requested comes from the "cloud" instead of a fixed physical object. This application program operates somewhere in the "cloud." The users do not need to know the specific location of the app, and they can get various services through network services. All resources in cloud computing system are gathered into a dynamic resource pool, which is 
provided to all clients in a multi-tenant mode to make sure that the services between users are isolated and free from interference. Neural network prediction method is a method to conduct economic forecast by using neural network. As most economic problems are nonlinear and neural network model has excellent non-linear fitting, people have attached more and more importance to predict these complex problems [5]. The scale of neural network structure affects the learning capacity and generalization ability of neural network. If the structure is too small, the learning capacity is not sufficient; and if it is too big, the generalization ability is weakened. Structural optimization is to enable neural network to learn a proper structure, which maintains the best generalization while meeting the need of learning. This paper based on cloud computing platform, adopts BP neural network of momentum-adaptive learning rate, and studies the economic management forecast and the optimization of resource allocation [6].

In October 2008, Ian Foster-the father of the Grid, hosted the 1st Cloud Computing and Applications Workshop (CCA'08). After that, all conferences about network computing have made cloud computing one of the themes, including Cluster 2009, International Symposium on Cluster Computing and the Grid (CCGrid 2009), and International Conference on Grid and Cooperative Computing (GCC 2009). Meanwhile, the cooperation between Google and University of Washington has launched the Academic Cloud Computing Initiative to promote the popularity and enhance the study of cloud computing. Back Propagation (BP) neural network is a multi-layer feedforward neural network based on error back propagation. Such error feedback network model uses the forward propagation of signal and the back propagation of error to dynamically adjust the weight and threshold between nodes in every layer so as to achieve the purpose of learning and training. BP neural network is a multi-layer forward neural network which has been applied most widely $[7,8]$. For a 3-layer BP neural network, one network with one hidden layer, it can approximate any continuous function in the bounded domain at any accuracy as long as there are sufficient hidden nodes. As the periodic fluctuation of macro-economy becomes increasingly obvious in economic operation, it is necessary to study and develop a framework to predict the future trend of economy or commodity [9]. At present, the frequently used economic forecasting system is operating on the centralized platform of a single micro-computer where different economic indicators use different prediction models and the results obtained are also different. Decision-makers do not know which prediction model to use for economic indicators to get the optimal result in advance; so they can only choose the model based on their own feeling and the result may not be the best. The combination of cloud computing and neural network can automatically combine prediction indicators and prediction models, predict every possibility, automatically simulate, and analyze the prediction result and the actual conditions so as to choose the best prediction. Then, the model and result are returned to the decision-makers [10, 11].

\section{Cloud-based economic forecast framework}

\subsection{Overall architecture}

Cloud-based economic forecast framework includes two levels, i.e., distributed computing layer and user interface layer. 
(1). Cloud client: mainly used to display the prediction result, including the prediction result table, the prediction result graph of actual curve, and the prediction curve fitting, for the decision-makers for make comparisons.

(2). Master server: mainly responsible for receiving requests from cloud client and decomposing a prediction task proposed by the client into multiple sub-tasks. It arranges and combines the economic indicators to be forecasted and all algorithms in the model table and then assigns every possible combination to an idle computational node. Then, the corresponding computational node proceeds with the prediction.

(3). Storage node cluster: made up of several computers with a huge storage function and mainly responsible for accessing digital resources and prediction model $[12,13]$.

(4). Computational node cluster: composed of several computers and mainly responsible for handling all computation involving economic indicators and specific prediction models. It can avoid the problems caused by the economic prediction system based on a single microcomputer. As far as the computer speed is concerned, it will acquire higher computing power than the common computers with no bottleneck occurring in the storage capacity $[14,15]$.

The development and change of economic phenomena are subject to various factors, which are internally connected. The accuracy of economic forecast has its relativity and restrictions. The growth and change of economic phenomena are factor not only by the factors that are already known to and under the control of people, but also the factors that people do not know and cannot control, e.g., major economic policies and major natural disasters which have a tremendous impact on economy. Therefore, the future development of economic phenomena is uncertain. The bias between economic prediction result and actual economic operation is not equal to the failure of prediction. By analyzing the result of economic forecast, government departments or individuals can make necessary adjustments and interventions to economic activities and adopt the corresponding economic measures to make economic activities go where harm can be avoided and lead to the development of economic activities. The forecast result does not coincide with the actual economic operations.

\subsection{Function analysis}

The function of this framework includes two parts. One is the maintenance function of the backend data. After logging in with authentication, the administrator can maintain the backend data. The maintenance of backend data includes the maintenance of economic indicators, data, and prediction model. Among them, index maintenance means that the administrator can check the existing indicators through the page when the system is operating and editing the existing indicators; add new indicators as required; and delete overdue indicators. Data maintenance is to store every value of indicator in the corresponding database. And model maintenance is to modify the existing prediction model in the system, add new model, and delete the old model. The other is the function of interface display, and it browses the prediction result of various indicators in the form of table or graph. In the prediction, the content includes the prediction of the major macro-economic indicators, and in the display platform, it includes the 
prediction result table, the prediction curve of actual curve, and the prediction curve fitting. In the analysis of the prediction result, it includes graphic and text description. Every indicator has many functions at the same time, e.g., the ability to inquire the prediction values in different periods of time and find the fitting of the corresponding prediction value.

Apart from a thorough knowledge of the history and status quo of the object to be forecasted, the accuracy of economic forecast also depends on the selection of scientific and rational forecasting methods, which rests with the in-depth understanding of economic activities and the mastery economic theories [16]. Qualitative forecasting method means that the predictor makes the judgment on the nature, direction, and extent of economic development according what they have mastered, their practical experience, and expertise. With qualitative forecast, suggestions can be made to provide evidence for the government and enterprises in formulated economic decisions, plan management, and guidance. Quantitative forecasting method uses quantitative knowledge and mathematical model to conduct quantitative measure on the future economic phenomena. This kind of method mainly includes time series prediction method, regression analysis prediction method, and tendency curve model prediction method [17].

\section{Method}

\subsection{Basic principle}

Learning, also known as training, is one of the most significant functions of neural networks. The input of network learning is usually a series of data, which are called as samples. As the input and output of network, the network trains the samples according to specific learning rules and learning algorithms. The structure of the connection strength between neurons and its correlated topology is adjusted in accordance with the relationship between the input and the output. In training, if the actual output of network meets the accuracy set by the modeler, the training model considers the training process is over; in another word, the learning model is success.

Neural network algorithm includes two steps: the forward propagation of work signal and the back propagation of error signal. In the forward propagation, it inputs the work signal from the input signal, transmits the output of the input layer to the hidden layer, and forms the output of the hidden layer by processing the hidden layer. The output of the output layer is the final result. The error is computed by comparing the result of the output layer with the expected output. This is the road to go forward. In this propagation, the weights of the input layer, the hidden layer, and the output layer are fixed.

The back propagation estimates the error of the previous layer by using the error of forward propagation so as to get error estimates of all other layers by carrying out back propagation on every layer. To change the internal state means to change the weight of every layer in neural network and the error of the neural network can satisfy specific requirements. After training, neural network model shows certain rules in the input and output.

\subsection{Optimization model of resource allocation}

In the actual operation of projects, different resource allocation plans will result in different completion time. Through the combinations of different resource allocations, the 
completion time calculated has neglected the unit of time, maybe hour, day, or month. The completion time of the project is closely related to its cost and profit. As the runup time and the time of duration of a project are different, the cost of resource allocation changes inevitably. On the contrary, during the off reason, the cost of resource allocation is low due to resource demand. This indicates that a shorter construction period does not necessarily mean a lower cost of the entire project and a more feasible project. Therefore, to take into consideration, the project duration and the optimization plan of project resource allocation have great significance.

The analysis based on different resource allocation plans and under-construction resource allocation cost is the method of machine learning, and it enables the system to summarize from historical data and extract the law of run-up time of different projects to the resource allocation cost under project duration. By this means, it can estimate the resource allocation costs under different time resource allocation schemes. It can be seen that the optimization model of resource allocation can correspond to two stages in machine learning: environment preparation and learning. The application of optimized resource allocation model is the implementation link in machine learning. Figure 1 is the diagram of resource optimization.

\subsection{Design of BP neural network based on momentum-adaptive learning rate}

Additional momentum makes BP network considers not only the impact of error on the gradient, but also the impact on the change trend in error curve while revising the weight. Without additional momentum, the network may be trapped in shallow local minimum and the use of additional momentum might miss these minimums [18, 19]. This method adds a value directly proportional to the change in the previous weight to the change of every weight based on back propagation and brings about new weight change according to back propagation.

The weight adjustment equation with the factor of additional momentum is as follows:

$$
\Delta w_{i j}(k+1)=(1-m c) \eta \delta_{i} p_{j}+m c \Delta w_{i j}(k)
$$

$$
\Delta b_{i}(k+1)=(1-m c) \eta \delta_{i}+m c \Delta b_{i}(k)
$$

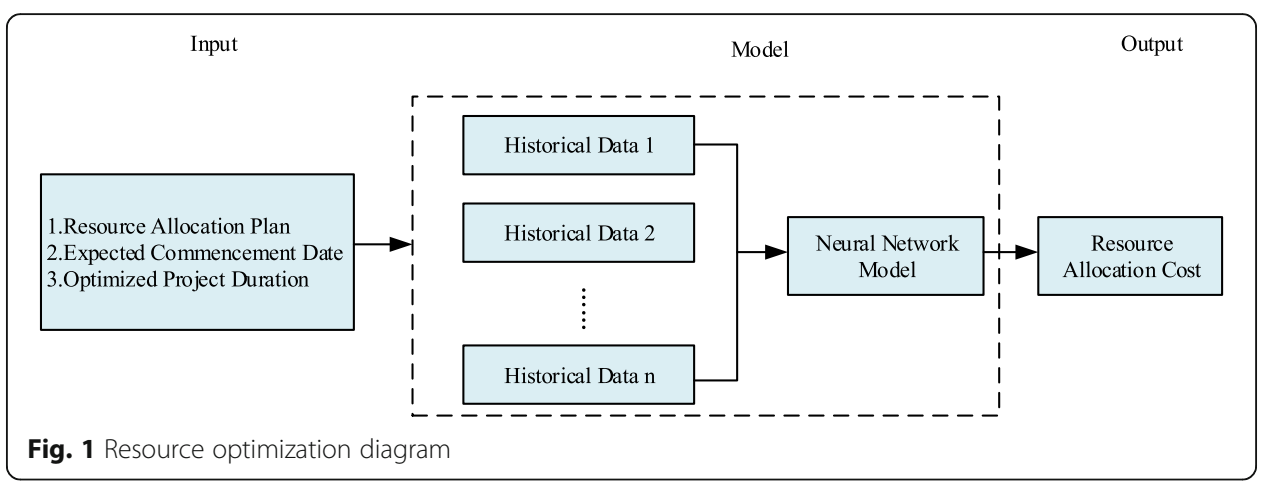


where $k$ is the number of training and $\mathrm{mc}$ is the factor of momentum, which is usually 0.95 .

In essence, additional momentum is to convey the impact of the last weight change through a factor of momentum [20,21]. When the value of the factor of momentum is ZERO, the weight changes according to the gradient descent algorithm and when it is ONE, the new weight change is the last weight change and the change part caused by gradient method will be ignored. In this way, when the item of momentum is added, it promotes the adjustment of weight to change toward the average direction of the bottom of error curve. When the network weight gets into the flat region of the bottom of error curve, $\delta_{i}$ will become very small and $\Delta w_{i j}(k+1)=\Delta w_{i j}(k)$ so as to avoid the emergence of $\Delta w_{i j}=0$ and enable the network to jump out of the local minimum of error curve.

In the design, the judgment condition to use momentum method is

$$
m c= \begin{cases}0 & E(k)>E(k-1) * 1.04 \\ 0.95 & E(k)<E(k-1) \\ m c & \text { Others }\end{cases}
$$

In which, $E(k)$ is the square sum of error in the $k$ thstep.

For a given problem, it is not an easy thing to choose the proper learning rate. Usually, it is obtained by experience or experiment; however, a good learning rate for the initial stage of the training does not mean a proper one in the follow-up training. In order to address this problem, it occurs to people to automatically adjust the learning rate in the training process. Generally, the criteria to adjust learning rate is to check whether the weight really lowers the error function. If so, it means that the learning rate chosen is small and a momentum can be added properly; if not, over-correction occurs and the value of learning rate shall be reduced. The adaptive learning rate is defined as follows:

$$
\eta(k+1)= \begin{cases}1.05 \eta(k) & E(k+1)<E(k) \\ 0.7 \eta(k) & E(k+1)>1.04 E(k) \\ \eta(k) & E(k+1)>1.04 E(k)\end{cases}
$$

where $E(k)$ is the error sum of squares. The value range of the initial learning rate $\eta(0)$ is very random.

When adopting additional momentum method, BP algorithm can find the global optimal solution and when using adaptive learning rate, BP algorithm can shorten the training time; therefore, this paper trains BP neural network with momentum-adaptive learning rate adjustment algorithm. The flowchart of BP neural network is shown as Fig. 2.

\section{Results and discussion}

\subsection{Data selection and analysis}

Regional gross domestic product (regional GDP) refers to the total final result of all units in this region to carry out production activities at a certain period of time (usually a year or a quarter) and the value of the labor. Regional GDP has great significance, 


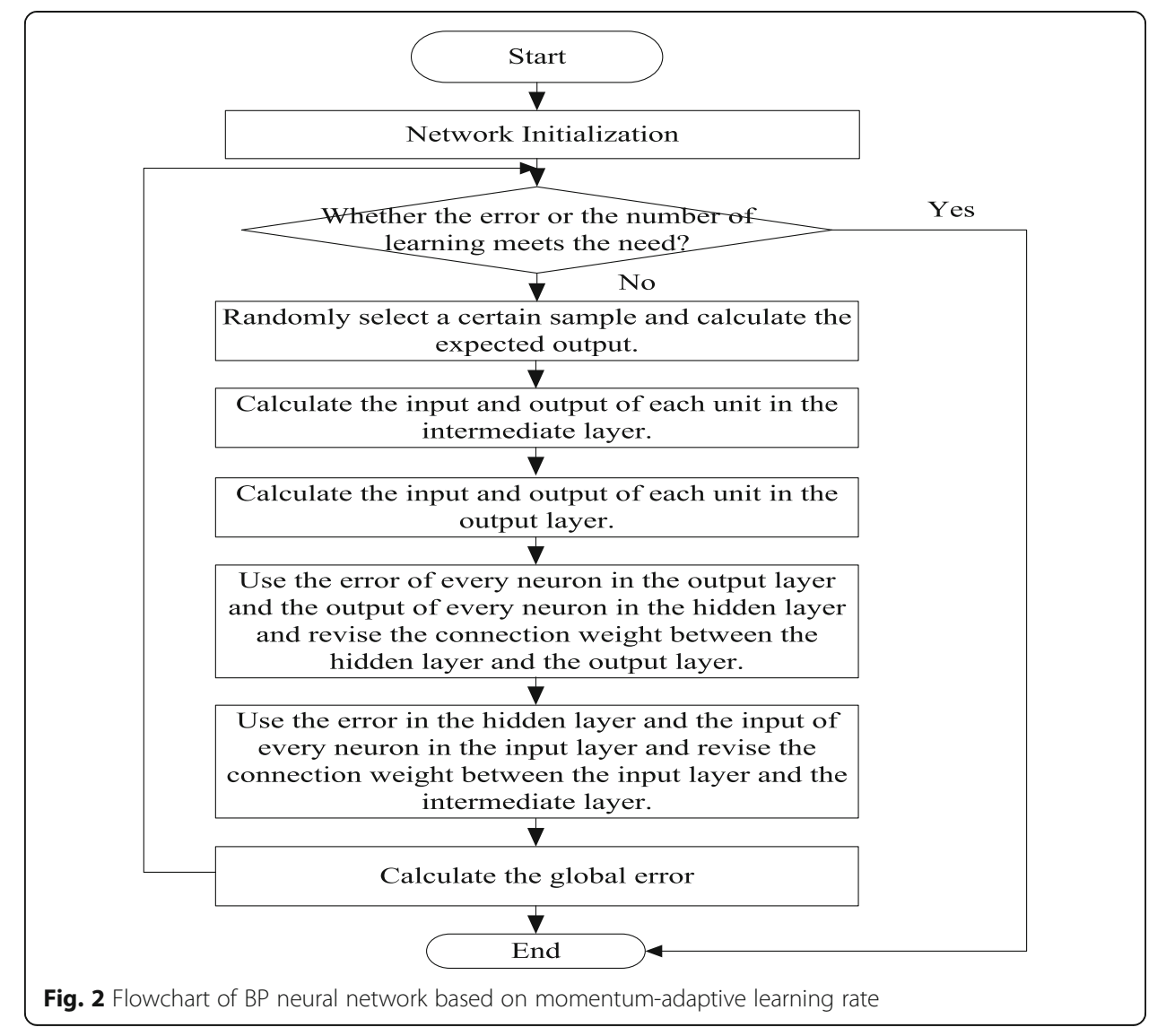

and it is deemed as a criterion to evaluate the development level and economic conditions of a region at this period of time. This paper collects valid data and uses Matlab software platform for BP neural network model simulation for a certain regional GDP.

We have chosen household consumption level, permanent population, average wage, registered urban unemployment rate, cargo volume, passenger volume, and data of medical and health institutions as the input samples of BP neural network and the regional GDP as the predicted output of the model.

\subsection{Result and analysis of experiment simulation}

\subsubsection{Layers of network}

It has bias and at least one S-shaped hidden layer and a linear output layer, and it can approximate any rational number. Increasing the layers can further reduce errors and improve accuracy, but meanwhile, it also makes the network more complex and increase the training time of network weight.

\subsubsection{Neurons in the hidden layer}

In order to avoid the "over-fitting" phenomena as many as possible and ensure sufficient network performance and generalization ability, the most fundamental principle to identify the number of nodes in the hidden layer is as follows: take as compact a 
structure as possible while meeting the accuracy demand, in other words, take as few nodes in the hidden layer as possible. Research shows that the number of nodes in the hidden layer is related to not only the nodes of the input/output layer, but also the factors such as the complexity of the problem to be solved, the type of the transfer function, and the property of sample data.

\subsubsection{Selection of initial weight}

As the system is nonlinear, the initial value is closely related to whether the learning can reach the local minimum, whether it can converge and the length of training time. Generally, it is hope that the output value of every neuron after initial weight is close to zero. In this way, the weight of each neuron is ensured that it is adjusted within the maximum change of their activation functions. So, the initial weight is a random number between $(-1,1)$.

\subsubsection{Learning rate}

Learning rate decides the change of weight generated in every cyclic training. A big learning rate may result in uncertain system, but a small one may lead to a longer training time and a slow convergence. So it tends to choose a small learning rate to ensure the stability of the system. The value range of learning rate is $(0.01,0.8)$.

According to the number of input data, the number of nodes in the input layer is 7 . According to the output data, the number of nodes in the output layer is set as 1 . The number of nodes in the hidden layer is 10 . The empirical equation for the number of nodes in the hidden layer is as follows:

$$
\begin{aligned}
& m=\sqrt{n+1}+\alpha \\
& m=\log _{2} n \\
& m=\sqrt{n l}
\end{aligned}
$$

In which, $m$ is the number of nodes in hidden layer, $n$ is the input layer, $l$ is the output layer, and $\alpha$ is constant from 1 to 10 .

The corresponding data to different influence factors have different orders of magnitude, which will affect the learning effect of BP neural network. So the data shall be processed to eliminate such impact and ensure accurate results. The common normalization is the normalized data between $(0,1)$, and the implementation method is

$$
X=\frac{X-X_{\min }}{X_{\max }-X_{\min }}
$$

where $X_{\max }$ is the maximum value of the influence factor in which data $X$ is located and $X_{\min }$ is the corresponding minimum value.

For this model, the results are as shown in Figs. 3 and 4 below after several trainings.

In Fig. 3, $X$-axis is the number of training and the $Y$-axis is the mean squared error (MSE). The curve shows the error change of the network model with the increase of trainings, and the dotted line reflects the error goal we set. It can be seen that after several trainings, the error has achieved the expected goal. As shown in Fig. 4, the functional relationship between the expected output and the actual 


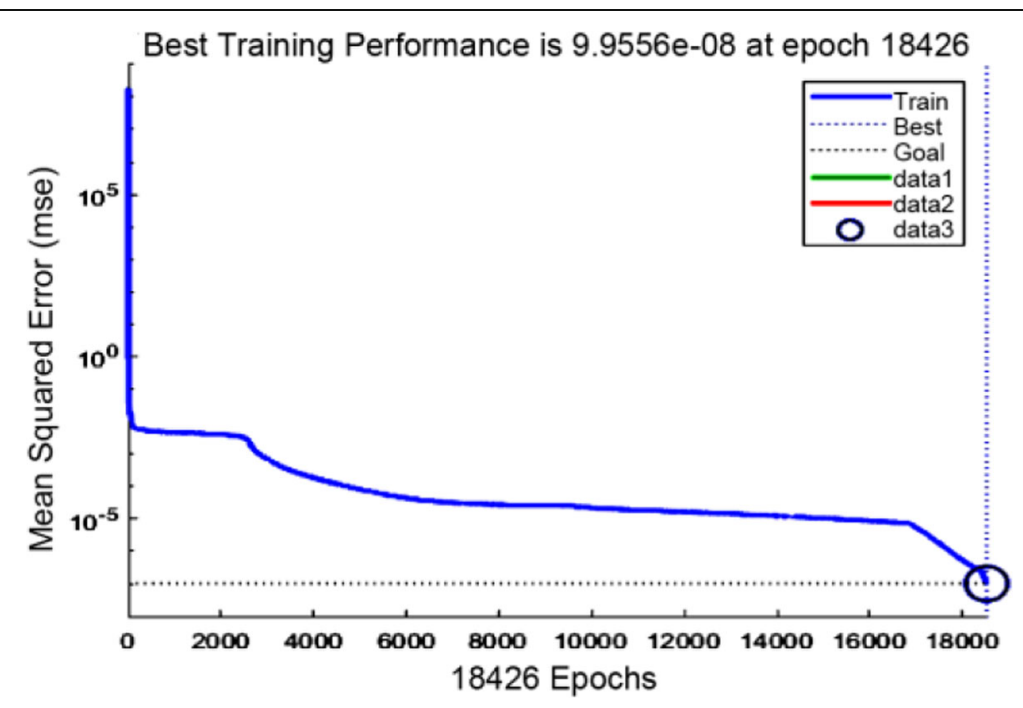

Fig. 3 Performance analysis

output basically fluctuates around a straight line with good fitting effect and ideal training effect. Figure 5 shows the change of gradient in the training process of the entire network. The prediction results are shown in the Table 1.

As can be seen from Table 1, the prediction accuracy is $87.34 \%$, and the average error is $12.66 \%$. The comparison results between the predicted value and the actual value are shown in Fig. 6.

In Fig. 6, the abscissa is the year and the ordinate is the output value. Among them, the output of the neural network is very close to the predicted output, and the predicted GDP is consistent with the actual GDP. The output result of neural network is very close to the expected output. The expected regional GDP basically coincides with the actual regional GDP, as demonstrated in the Fig. 7 and Fig. 8.

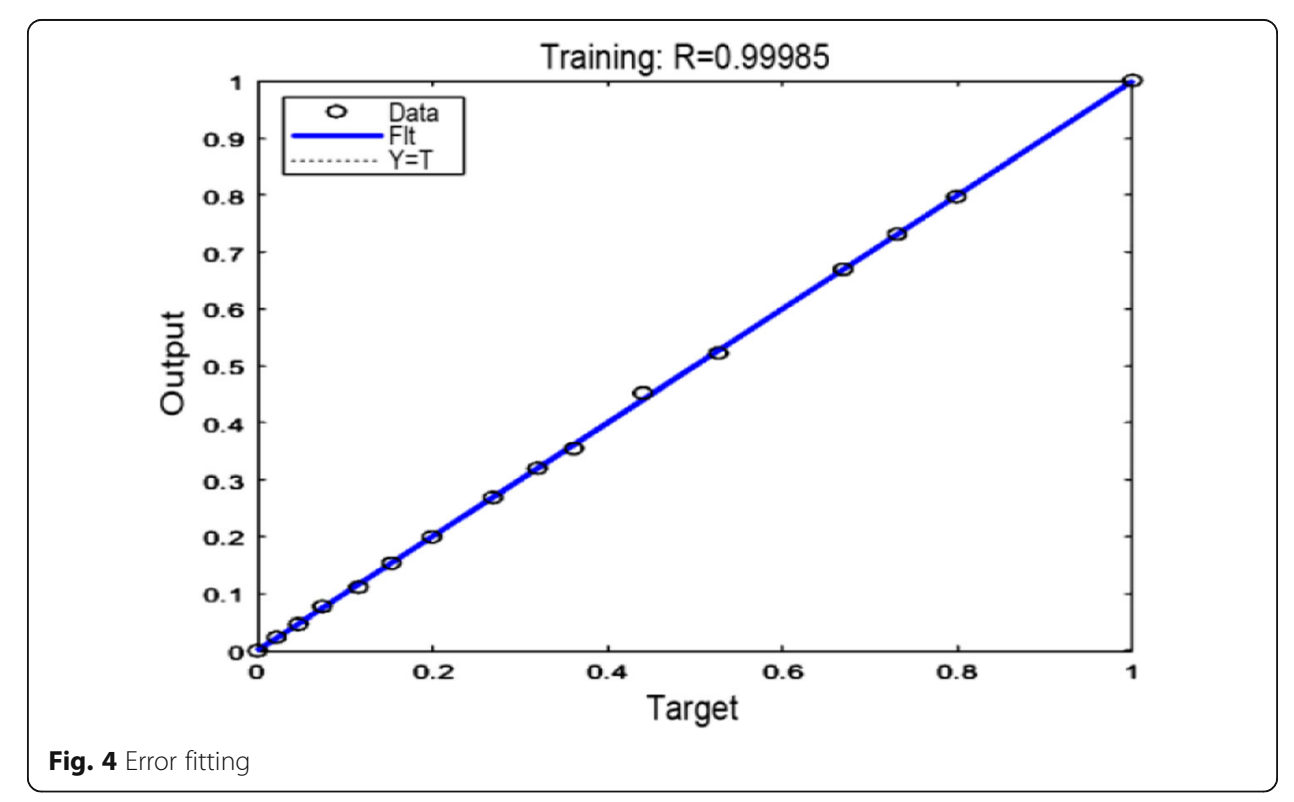




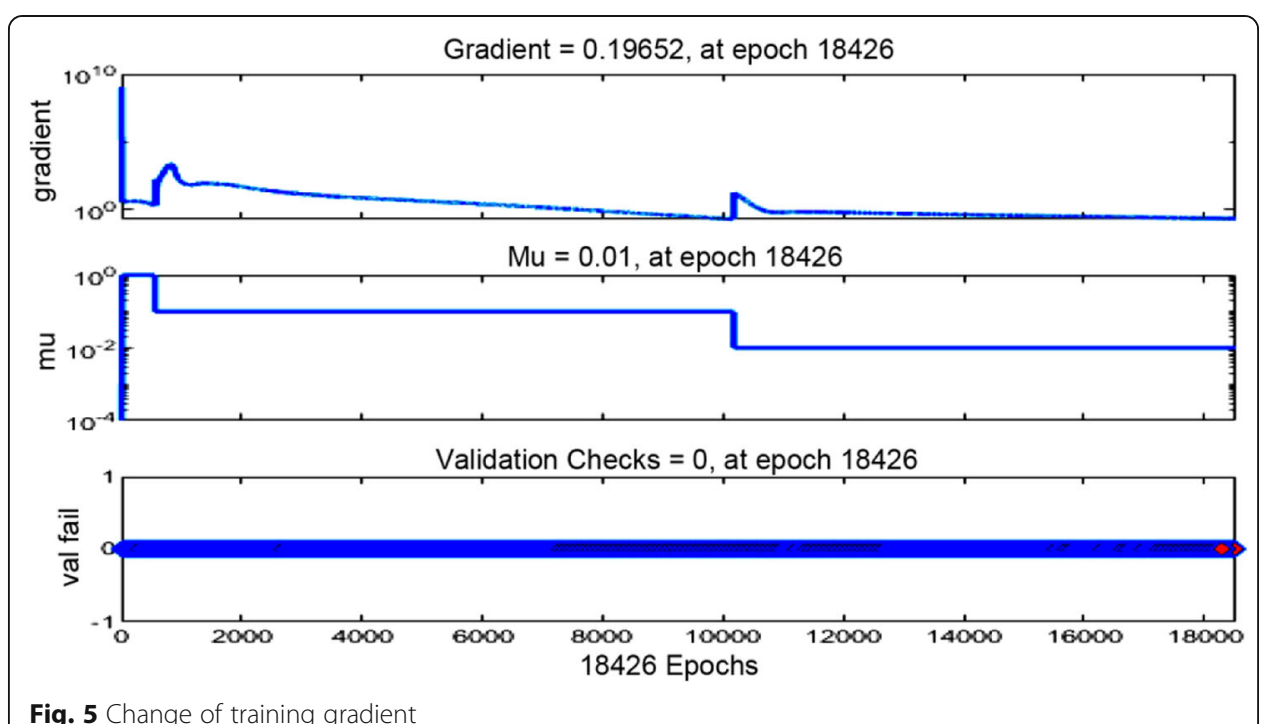

Fig. 5 Change of training gradient

After training, we have got the necessary network model, according to the design principle of additional momentum method, when a revised weight leads to a too big increase result in the error, the new weight shall be canceled and shall not be adopted and the role of momentum shall be stopped so as to prevent the network from entering big error curved surface. When the new change rate of error exceeds the previously set maximum change rate of error, the change of weight calculated shall also be canceled. The maximum change rate of error can be any value bigger than or equal to 1 . In BP neural network training of additional momentum method, conditions shall be added to determine the use of its weight revision equation correctly.

Table 1 The prediction results

\begin{tabular}{llllll}
\hline Year & $\begin{array}{l}\text { Real value (RMB 100 } \\
\text { million) }\end{array}$ & $\begin{array}{l}\text { Predicted value (RMB 100 } \\
\text { million) }\end{array}$ & $\begin{array}{l}\text { Error value (RMB 100 } \\
\text { million) }\end{array}$ & $\begin{array}{l}\text { Accuracy } \\
(\%)\end{array}$ & $\begin{array}{l}\text { Error rate } \\
(\%)\end{array}$ \\
\hline 2000 & 2975.63 & 3399.66 & 424.03 & 85.75 & 14.25 \\
2001 & 3528.81 & 3198.51 & 330.30 & 90.64 & 9.36 \\
2002 & 4633.56 & 5169.66 & 536.10 & 88.43 & 11.57 \\
2003 & 5236.17 & 4823.04 & 413.13 & 92.11 & 7.89 \\
2004 & 5941.82 & 5191.96 & 749.86 & 87.38 & 12.62 \\
2005 & 7069.34 & 7996.13 & 926.79 & 86.89 & 13.11 \\
2006 & 8308.25 & 6834.37 & 1473.88 & 82.26 & 17.74 \\
2007 & 9934.37 & 8757.15 & 1177.22 & 88.15 & 11.85 \\
2008 & 11595.68 & 10401.32 & 1194.36 & 89.7 & 10.3 \\
2009 & 12542.23 & 14428.58 & 1886.35 & 84.96 & 15.04 \\
2010 & 14206.45 & 12263.01 & 1943.44 & 86.32 & 13.68 \\
2011 & 16020.8 & 17545.98 & 1525.18 & 90.48 & 9.52 \\
2012 & 17658.34 & 20236.46 & 2578.12 & 85.4 & 14.6 \\
2013 & 18842.2 & 21274.73 & 2432.53 & 87.09 & 12.91 \\
2014 & 20431.66 & 23588.35 & 3156.69 & 84.55 & 15.45 \\
\hline
\end{tabular}




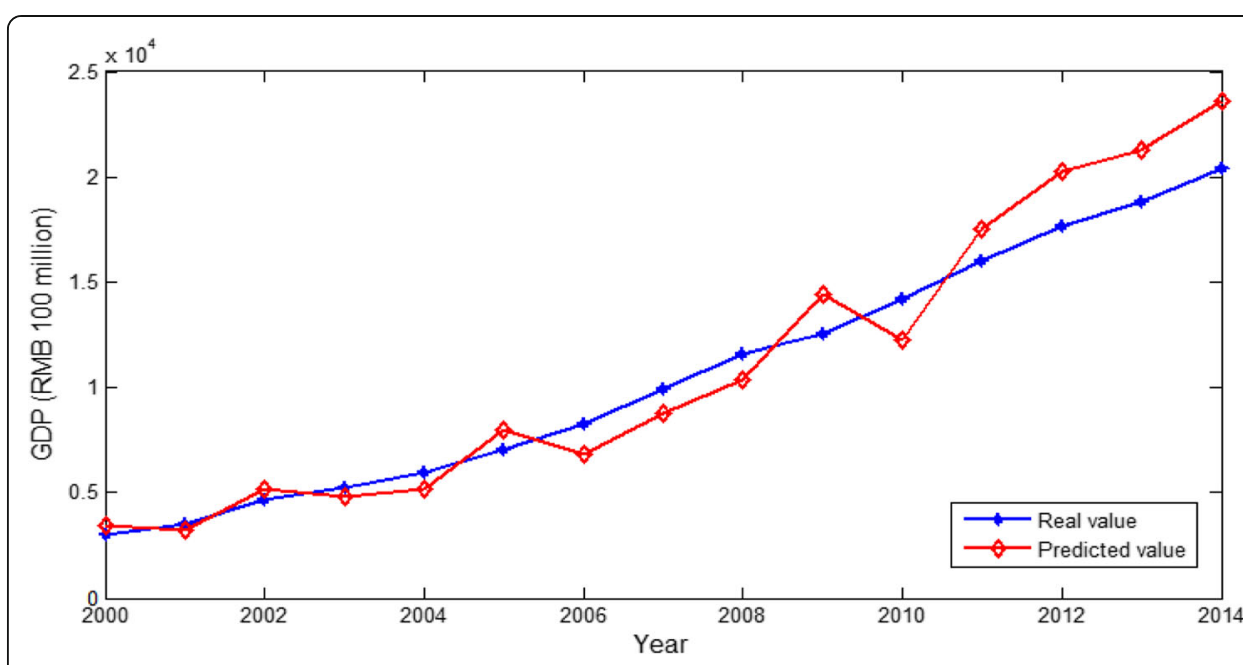

Fig. 6 Comparison between predicted value and real value

\section{Conclusion}

Cloud computing has many strengths, e.g., convenience, economy, high expansibility, and availability. Cloud computing environment can dynamically schedule resources and users can subscribe the corresponding resources based on their actual needs and the environment can also make adjustments at any time to cope with rapid changes when user needs change. Artificial neural networks can process parallel, learn automatically, and approximate any nonlinear functions at any accuracy, and it is suitable for modeling of complex systems and predictive analysis. This paper is based on cloud computing platform, and it adopts momentum-adaptive learning rate BP neural network, and studies economic management forecasts and resource allocation optimization. The simulation experiment has proven that the

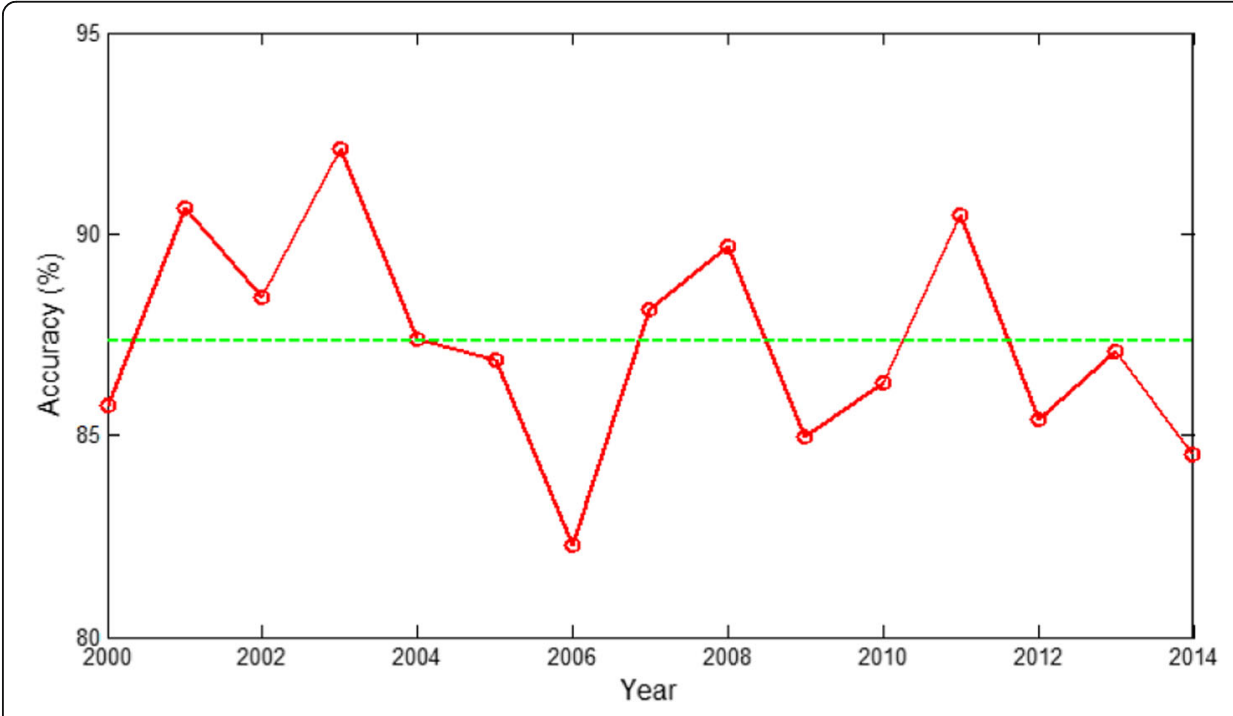

Fig. 7 Network accuracy 


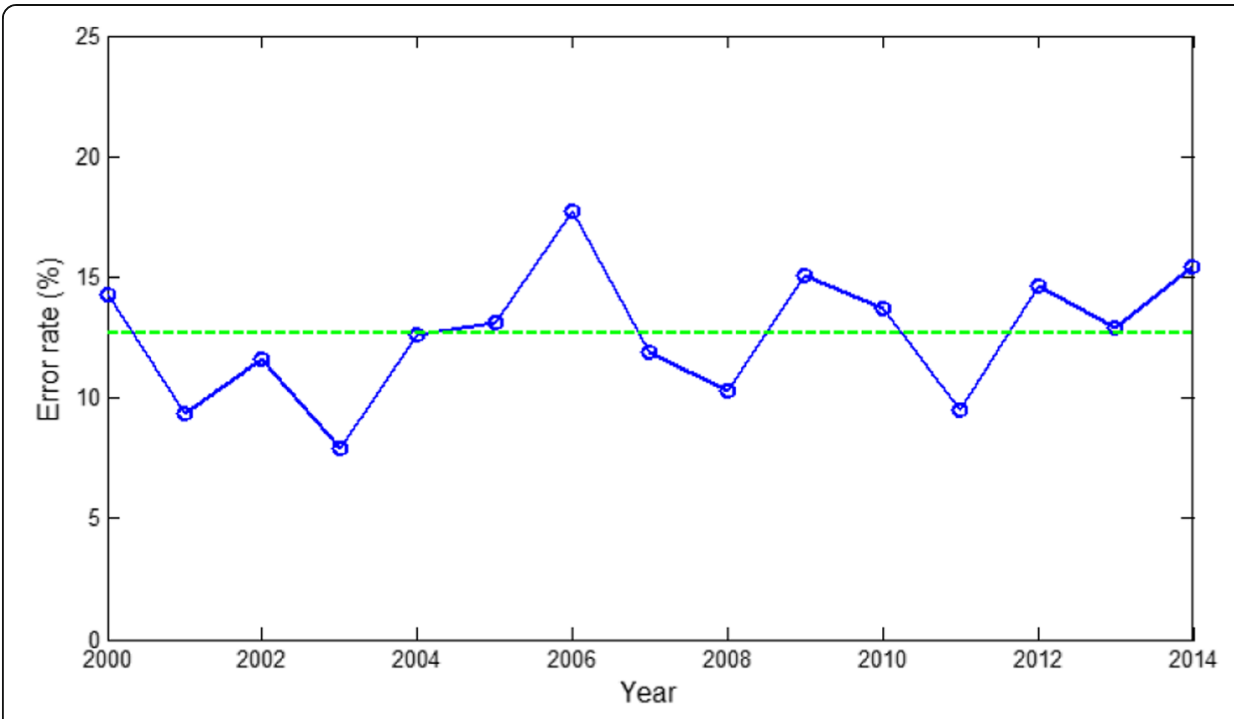

Fig. 8 Network prediction error ratio

proposed algorithm has the following advantages: (1) it has strong optimized computing power, (2) it does not rely on gradient information, (3) it is applicable for solving highly complex non-linear problems, (4) it can conduct analysis and forecasts for the economic management, and it can provide strong decision support for decision makers.

\section{Acknowledgements}

The authors acknowledged the anonymous reviewers and editors for their efforts in valuable comments and suggestions.

\section{Author's contributions}

$\mathrm{PH}$ proposes the innovation ideas and drafts the manuscript. The author(s) read and approved the final manuscript.

\section{Funding}

Funding is not applicable

Availability of data and materials

Data sharing is not applicable to this article as no datasets were generated or analyzed during the current study.

\section{Competing interests}

The authors declare that they have no competing interests.

Received: 23 May 2020 Accepted: 14 August 2020

Published online: 27 August 2020

\section{References}

1. L. Falat, L. Pancikova, Quantitative modelling in economics with advanced artificial neural networks. Proced Econ Finance 34(1), 194-201 (2015)

2. N. Gupta, S. Nigam, Crude oil price prediction using artificial neural network. Proced Comput Sci 170(1), 642-647 (2020)

3. N.E.G.A.S.H. Berihun Mamo, Y.A.W. Atta Dennis, Artificial neural network based production forecasting for a hydrocarbon reservoir under water injection. Petrol Explor Dev 47(2), 383-392 (2020)

4. A.E. Tümer, A. Akkuş, Forecasting gross domestic product per capita using artificial neural networks with noneconomical parameters. Physica A 51, 468-473 $(215,2018)$

5. M. Zhang, D. Zhang, H. Yao, et al., A probabilistic model of human error assessment for autonomous cargo ships focusing on human-autonomy collaboration. Safety Sci 130(1), 104838 (2020)

6. L.-Y. Leong, T.-S. Hew, K.-B. Ooi, G.W.-H. Tan, Predicting actual spending in online group buying - an artificial neural network approach. Electron Commerce Res Appl 38(11), 100898 (2019)

7. Zhenhua Huang, Xin $\mathrm{Xu}$, Juan $\mathrm{Ni}$, Honghao Zhu, Cheng Wang. Multimodal representation learning for recommendation in Internet of Things. IEEE Int Things J 6(6), 10675-10685(2019).

8. H. Hao, Q. Zhang, Z. Wang, J. Zhang, Forecasting the number of end-of-life vehicles using a hybrid model based on grey model and artificial neural network. J Cleaner Prod 202(20), 684-696 (2009) 
9. J. Hu, Y. Sun, G. Li, G. Jiang, B. Tao, Probability analysis for grasp planning facing the field of medical robotics. Measurement 141(1), 227-234 (2019)

10. N. Silva, L.M.D.F. Ferreira, C. Silva, V. Magalhães, P. Net, Improving supply chain visibility with artificial neural networks, Proced Manufact 11(1), 2083-2090 (2017)

11. T.H. Nguyen, D. Nong, K. Paustian, Surrogate-based multi-objective optimization of management options for agricultural landscapes using artificial neural networks. Ecol Model 400(24), 1-13 (2019)

12. D. Jiang, G. Li, Y. Sun, J. Kong, B. Tao, Gesture recognition based on skeletonization algorithm and CNN with ASL database. Multimedia Tools Appl 78(21), 29953-29970 (2019)

13. H. Ghoddusi, G.G. Creamer, N. Rafizadeh, Machine learning in energy economics and finance: a review. Energy Econ 81(6), 709-727 (2019)

14. T. Nguyen-ky, S. Mushtaq, A. Loch, K. Reardon-Smith, T. Tran-Cong, Predicting water allocation trade prices using a hybrid artificial neural network-Bayesian modelling approach. J Hydrol 567(12), 781-791 (2018)

15. F. Hu, G. Wu, Distributed error correction of EKF Algorithm in multi-sensor fusion localization model. IEEE Access 8(1), 93211-93218 (2020)

16. Z. Yang, D.K.J. Lin, A. Zhang, Interval-valued data prediction via regularized artificial neural network. Neurocomputing $331(28), 336-345(2019)$

17. W. Wei, X. Xia, W. Marcin, X. Fan, R. Damasevicius, Y. Li, Multi-sink distributed power control algorithm for cyber-physicalsystems in coal mine tunnels. Comput Netw 161(1), 210-219 (2019)

18. L. Dong, Q. Guo, W. Wu, Speech corpora subset selection based on time-continuous utterances features. J Combinatorial Opt 37(4), 1237-1248 (2019)

19. F. Long, N. Xiong, A.V. Vasilakos, L. Yang, F. Sun, A sustainable heuristic QoS routing algorithm for pervasive multilayered satellite wireless networks. Wireless Netw 16(6), 1657-1673 (2010)

20. J. Li, N. Xiong, J.H. Park, C. Liu, M.A. Shihua, S.E. Cho, Intelligent model design of cluster supply chain with horizontal cooperation. J Intelligent Manufact 23(4), 917-931 (2012)

21. W. Wei, X. Fan, H. Song, X. Fan, J. Yang, Imperfect information dynamic stackelberg game based resource allocation using hidden Markov for cloud computing. IEEE Trans Serv Comput 11(1), 78-89 (2016)

\section{Publisher's Note}

Springer Nature remains neutral with regard to jurisdictional claims in published maps and institutional affiliations.

\section{Submit your manuscript to a SpringerOpen ${ }^{\circ}$ journal and benefit from:}

- Convenient online submission

- Rigorous peer review

- Open access: articles freely available online

High visibility within the field

- Retaining the copyright to your article

Submit your next manuscript at $\boldsymbol{\nabla}$ springeropen.com 